\title{
Factors associated with stereopsis and a good visual acuity outcome among children in the Infant Aphakia Treatment Study
}

SR Lambert ${ }^{1}$, L DuBois ${ }^{1}, \mathrm{G}$ Cotsonis², EE Hartmann ${ }^{3}$ and C Drews-Botsch ${ }^{4}$ for the Infant Aphakia Treatment Study Group ${ }^{5}$

\begin{abstract}
Purpose To identify factors associated with stereopsis in children with good visual acuity after unilateral congenital cataract surgery in the Infant Aphakia Treatment Study.

Patients and methods Infants with a unilateral congenital cataract $(n=114)$ were randomized to IOL implantation or contact lens correction after cataract surgery. At age 4.5 years, a masked examiner assessed HOTV acuity and stereopsis. Adherence to patching was assessed using 48-h recall telephone interviews and 7-day diaries throughout the first 5 years of life. Ocular motility was evaluated at age 5 years. Baseline, postoperative, and adherence findings were compared between patients with $20 / 40$ or better acuity in their treated eyes with or without stereopsis.

Results Thirty (27\%) of 112 patients who were evaluated at age 4.5 years had $20 / 40$ or better acuity in their treated eye. Stereopsis was present on one or more tests in $\mathbf{1 5}$ of these $30(50 \%)$ children. Baseline characteristics and postoperative findings did not differ between patients with or without stereopsis. Children with stereopsis were more likely to be orthotropic at distance $(P=0.003)$ and were patched for fewer hours per day throughout the first 5 years of life and the difference increased over time $(P<0.001)$. On average children with stereopsis were patched for $3.4 \mathrm{~h} /$ day during the first year of life and patching steadily decreased to $1.8 \mathrm{~h}$ /day by age 4 years. Conclusion Among children with good vision following unilateral congenital cataract surgery, orthophoria and fewer hours of patching, particularly during years 2,3 , and 4 , are associated with some evidence of stereopsis.
\end{abstract}

Eye (2016) 30, 1221-1228; doi:10.1038/eye.2016.164; published online 29 July 2016

\section{Introduction}

Fifty years ago it was believed that it was almost impossible for an eye with a unilateral congenital cataract to achieve good visual acuity. ${ }^{1-3}$ However beginning in the 1970s, small case series reported 20/40 or better acuity in the treated eyes of children with unilateral congenital cataracts after early cataract surgery coupled with contact lens wear and patching therapy. ${ }^{4,5}$ Birch and Stager ${ }^{6}$ have emphasized the importance of early cataract surgery (6 weeks or younger) to achieve a good visual outcome. In the Infant Aphakia Treatment Study (IATS), we found that socioeconomic status, measured by the availability of private insurance, and greater amounts of patching were the best predictors of a good visual acuity outcome. ${ }^{7,8}$ However, neither variable accounted for $>15 \%$ of the variance in visual outcomes.

In recent years, there have been reports that stereopsis is present in a small subset of children after unilateral congenital cataract surgery. ${ }^{9-11}$ We have previously reported a positive stereopsis response to at least one stereopsis test at age 4.5 years in $25 \%$ of children in the IATS cohort. ${ }^{12}$ We reported that positive stereopsis responses occurred more often in children who underwent cataract surgery at a younger age, had a better visual outcome, and were orthophoric at age 5 years. ${ }^{12,13}$ The purpose of this study was to identify baseline factors, postoperative findings, and treatment compliance factors associated with both good visual acuity and stereopsis. Our hope was that by identifying these factors that treatment protocols would be modified to allow a higher
${ }^{1}$ Department of Ophthalmology, Emory Eye Center, Emory University School of Medicine, Atlanta, GA, USA

${ }^{2}$ Department of Biostatistics and Bioinformatics, Atlanta, GA, USA

${ }^{3}$ University of Alabama, Birmingham, AL, USA

${ }^{4}$ Department of Epidemiology, Rollins School of Public Health, Emory University, Atlanta, GA, USA

Correspondence:

SR Lambert, Department of Ophthalmology, Emory Eye Center, Emory University School of Medicine, 1365-B Clifton Road, Atlanta, GA 30322, USA Tel: +1 404778 4417; Fax: +1 4047785203 . E-mail: slamber@emory.edu

${ }^{5}$ Members of the Infant Aphakia Treatment Study Group are listed before References.

Received: 8 June 2016 Accepted in revised form: 1 July 2016 Published online: 29 July 2016 
percentage of children to achieve nearly normal visual function after unilateral congenital cataract surgery.

\section{Materials and methods}

The study design, surgical techniques, follow-up schedule, patching and optical correction regimens, evaluation methods, and baseline patient characteristics have been reported previously and are only summarized in this report. ${ }^{14-16}$ This study was approved by the institutional review boards at all participating institutions and was in compliance with the Health Insurance Portability and Accountability Act. This study was supported through a cooperative agreement with the National Eye Institute of the National Institutes of Health and is registered with ClinicalTrials.gov.

The main inclusion criteria were a visually significant congenital cataract ( $\geq 3 \mathrm{~mm}$ central opacity) in one eye and an age of 28 to $<210$ days at the time of cataract surgery. Patients were randomized to have either an IOL implanted at the time of cataract surgery or to be left aphakic and corrected with a contact lens.

\section{Patching regimen}

Starting the second postoperative week, parents were instructed to have their child wear an adhesive occlusive patch over the unoperated eye for $1 \mathrm{~h}$ per day for each month of age until age 8 months. Thereafter, patching was prescribed for one-half of waking hours either by patching all waking hours every other day or for one-half of the patient's waking hours every day.

\section{Adherence to patching and optical correction}

Adherence to patching and optical correction was assessed using 48-h recall telephone interviews and 7-day diaries. ${ }^{8}$ The interviews were conducted every 3 months starting 3 months after surgery. Caregivers completed a 7-day patching diary 2 months after surgery and annually thereafter ( 1 month after the child's birthday). We calculated the mean proportion of waking time the optical correction was worn at each assessment. For the present analyses, we calculated the mean hours of daily patching and proportion of prescribed patching reported for all assessments within five 12-month periods: birth to 12 months of age, 12 to $<24$ months, 24 to $<36$ months, 36 to $<48$ months, and 48 to $<60$ months of age. For each time period, we restricted the analyses to children with at least three adherence assessments.

\section{Visual acuity and stereopsis assessment}

At age 4.5 years, a masked traveling examiner assessed monocular optotype acuity using the ATS-HOTV test and stereopsis using three different tests: (1) Frisby Stereotest (Richmond Products, Albuquerque, NM, USA), (2) Randot Preschool Test (Stereo Optical, Chicago, IL, USA), and (3) Titmus Fly Test (Stereo Optical, Chicago, IL, USA). ${ }^{16}$ A more detailed description of the procedures used for testing stereopsis have been published previously. ${ }^{12}$

\section{Ocular motility testing}

Ocular alignment was assessed at age 5 years at near and distance by an IATS-certified investigator using procedures that have been described previously. ${ }^{13}$ Investigators were instructed to measure any deviation in the appropriate optical correction. The timing and choice of strabismus surgery was at investigator discretion.

\section{Statistical considerations}

Difference in baseline characteristics and postoperative factors were compared between patients with and without stereopsis using $\chi^{2}$ tests, Fisher's exact tests, and independent group $t$-tests. Difference between patients with and without stereopsis for contact lens compliance, spectacle wear compliance, and hours of daily patching were analyzed using 95\% confidence intervals derived from independent group $t$-tests. All reported $P$-values are twosided, without adjustment for multiple testing; $P$-values of 0.05 or less were considered statistically significant.

\section{Results}

Thirty of the 112 (28\%) participants in the IATS who had their optotype acuity measured at age 4.5 years had $20 / 40$ or better acuity in their treated eye. The fellow eyes for these 30 patients all had 20/32 or better acuity except for 1 patient with 20/32 acuity in the treated eye and $20 / 50$ acuity in the fellow eye (when retested at age 5 years acuity was 20/32 in both eyes).

Stereopsis was present in 15 of 30 (50\%) patients with $20 / 40$ or better visual acuity. ${ }^{12}$ Twelve patients had $3000 \mathrm{~s} /$ arc of stereopsis using the Titmus test, 8 had 170 or $340 \mathrm{~s} /$ arc using the Frisby test and 4 had 400 or 800 s/arc using the Randot preschool test. However, only three patients had measureable stereopsis using all three tests and an additional four patients using two tests.

Cataract surgery was performed at a median age of 35 days in patients with stereopsis and 43 days in patients without stereopsis $(P=0.13)$. Although more of the children with stereopsis underwent cataract surgery 
when 4-6 weeks of age, the difference was not statistically significant $(P=0.27)$ (Table 1$)$. Mean corneal diameter and axial length were similar in both groups. We analyzed a variety of demographic factors including race, type of medical insurance, and gender, and did not find that any of these factors were associated with the likelihood of developing stereopsis. Postoperative factors such as type of optical correction, adverse events, additional intraocular surgeries, glaucoma, visual acuity, refraction, and the absolute prediction error in pseudophakic eyes were also not significantly related to the probability of developing measurable stereopsis (Table 2). More children with stereopsis were orthotropic at age 5 years compared with children without stereopsis (10/15 (67\%) vs $2 / 15(13 \%) ; P=0.003)$ and fewer children with stereopsis had undergone strabismus surgery compared with children without stereopsis. As it has been reported that true stereopsis cannot be present in patients with $\geq 6$ prism diopters (PD) of strabismus, ${ }^{17}$ we reviewed the angle of strabismus in the five patients with stereopsis who were not orthotropic. One child had a small esotropia when looking through the top half of his spectacles, but was orthotropic when looking through his bifocal. A second child only had a 3 PD esotropia at near. However, the other three patients had angles of strabismus $\geq 6$ PD (14 PD esotropia, 12 PD exotropia, and 18 PD exotropia). In addition, all three of these patients

Table 1 Baseline characteristics of patients with 20/40 or better acuity in treated eyes

\begin{tabular}{|c|c|c|c|}
\hline Factor & $\begin{array}{c}\text { Stereopsis } \\
(\mathrm{n}=15)\end{array}$ & $\begin{array}{c}\text { No stereopsis } \\
(\mathrm{n}=15)\end{array}$ & P-value \\
\hline Mean age at surgery (days) & $54 \pm 43$ & $74 \pm 50$ & 0.24 \\
\hline \multicolumn{4}{|l|}{ Age (weeks) } \\
\hline $4-6$ & $10(67 \%)$ & $7(47 \%)$ & 0.27 \\
\hline$>6$ & $5(33 \%)$ & $8(53 \%)$ & \\
\hline Mean corneal diameter $(\mathrm{mm})$ & $10.4 \pm 0.7$ & $10.5 \pm 0.6$ & 0.84 \\
\hline Mean axial length (mm) & $17.7 \pm 1.2$ & $18.2 \pm 1.3$ & 0.32 \\
\hline \multicolumn{4}{|l|}{ Type of cataract } \\
\hline Nuclear & $6(40 \%)$ & $7(47 \%)$ & 0.71 \\
\hline Non-nuclear & $9(60 \%)$ & $8(53 \%)$ & \\
\hline \multicolumn{4}{|l|}{ Gender } \\
\hline Male & $10(67 \%)$ & $5(33 \%)$ & 0.07 \\
\hline Female & $5(33 \%)$ & $10(67 \%)$ & \\
\hline \multicolumn{4}{|l|}{ Race } \\
\hline White & $12(80 \%)$ & $14(93 \%)$ & 0.60 \\
\hline Non-white & $3(20 \%)$ & $1(7 \%)$ & \\
\hline \multicolumn{4}{|l|}{ Private insurance } \\
\hline Yes & $10(67 \%)$ & $14(93 \%)$ & 0.07 \\
\hline No & $5(33 \%)$ & $1(7 \%)$ & \\
\hline
\end{tabular}

only had a stereopsis response to 1 of the 3 stereopsis tests (1 Frisby, 1 Titmus, and 1 Randot).

There was no evidence to suggest that the type of cataract was associated with the probability of developing stereopsis when good visual acuity was present. Both groups had nearly the same number of patients with nuclear (stereopsis group, $n=6$; no stereopsis group, $n=7$ ) and PFV (stereopsis group, $n=3$; no stereopsis group, $n=4$ ) cataracts. While more patients in the stereopsis group $(n=5)$ had posterior lentiglobus compared with the no stereopsis group $(n=2)$, the difference was not significant $(P=0.20)$. In addition, one patient in the no stereopsis group had a total cataract and one patient in both groups had other types of cataracts.

Among children randomized to contact lens wear who achieved good visual acuity, the average percentage of waking hours spent wearing a contact lens was similar for children with or without stereopsis (Table 3).

Table 2 Postoperative factors for patients with 20/40 or better acuity with or without stereopsis

\begin{tabular}{llll}
\hline Factor & $\begin{array}{l}\text { Stereopsis } \\
(\mathrm{n}=15)\end{array}$ & $\begin{array}{l}\text { No stereopsis } \\
(\mathrm{n}=15)\end{array}$ & P-value \\
\hline $\begin{array}{l}\text { LogMAR acuity } \\
\text { Treated eye }\end{array}$ & $0.18 \pm 0.08$ & $0.24 \pm 0.13$ & 0.13 \\
$\quad$ Fellow eye & $0.07 \pm 0.10$ & $0.10 \pm 0.12$ & 0.52 \\
& & & \\
$\begin{array}{l}\text { Optical correction } \\
\text { Contact lens }\end{array}$ & $7(47 \%)$ & $10(67 \%)$ & 0.23 \\
IOL & $8(53 \%)$ & $5(33 \%)$ & \\
& &
\end{tabular}

Last refraction

Contact lens $\quad+13.79 \pm 3.02+12.19 \pm 7.230 .59$

(D) IOL $\quad-2.95 \pm 3.75-1.70 \pm 3.340 .56$

Absolute prediction error in $1.80 \pm 1.68 \mathrm{D} \quad 1.52 \pm 0.86 \mathrm{D} \quad 0.74$

pseudophakic eyes

Adverse event(s)

$\begin{array}{llll}\text { Yes } & 12(80 \%) & 12(80 \%) & 1.00 \\ \text { No } & 3(20 \%) & 3(20 \%) & \end{array}$

Additional intraocular surgery

$\begin{array}{llll}\text { Yes } & 6(40 \%) & 5(33 \%) & 0.70\end{array}$

No $\quad 9(60 \%) \quad 10(67 \%)$

Glaucoma

$\begin{array}{llll}\text { Yes } & 0(0 \%) & 2(13 \%) & 0.48\end{array}$

No $\quad 15(100 \%) \quad 13(87 \%)$

Strabismus surgery

$\begin{array}{llll}\text { Yes } & 1(7 \%) & 9(60 \%) & 0.002\end{array}$

No $14(93 \%) \quad 6(40 \%)$

Orthophoric at age 5 years

$\begin{array}{llll}\text { Yes } & 10(67 \%) & 2(13 \%) & 0.003\end{array}$

No $5(33 \%) \quad 13(87 \%)$ resolution. 
While children randomized to receive an IOL who achieved good visual acuity and who had stereopsis wore spectacles fewer hours per day than children who had no evidence of stereopsis, the difference did not reach statistical significance (0.058) (Table 4). Overall, spectacle wear adherence was lower compared with contact lens wear adherence in both groups. This is not unexpected as some pseudophakic patients had a refractive error close to emmetropia.

Among children with visual acuity of $20 / 40$ or better, those with evidence of stereopsis tended to be patched for fewer mean hours per day compared with children with $20 / 40$ or better acuity without stereopsis (Table 5). Children with stereopsis were patched the greatest number of mean hours per day during the first year of life $(3.44 \pm 1.28 \mathrm{~h}$ per day); patching steadily declined thereafter to $1.79 \pm 1.73 \mathrm{~h}$ per day by age 4 years.
In contrast, children without stereopsis were patched the fewest number of mean hours per day during the first year of life ( $4.81 \pm 1.16 \mathrm{~h}$ per day) and patching increased thereafter to a high of $6.25 \pm 1.48 \mathrm{~h}$ per day at age 3 years. Although there was a small reduction in patching in this group at age 4 years, they were still being patched a mean of $5.89 \pm 1.44 \mathrm{~h}$ per day, which was $>4 \mathrm{~h}$ per day longer than children with stereopsis.

\section{Discussion}

We found that $27 \%$ of infants who underwent unilateral congenital cataract surgery in the IATS had 20/40 or better acuity in their treated eye at age 4.5 years and onehalf of these children had a stereopsis response on at least one test of stereopsis. Orthotropia and reduced patching, particularly during years 2, 3, and 4, both correlated with

Table 3 Contact lens compliance for treated eyes with 20/40 or better visual acuity with or without stereopsis

\begin{tabular}{lccccccc}
\hline Age & \multicolumn{2}{c}{ Stereopsis } & & \multicolumn{2}{c}{ No stereopsis } & Difference in compliance (\%) & 95\% CI of the difference (\%) \\
\cline { 2 - 3 } & No. of eyes & Compliance (\%) & & No. of eyes & Compliance (\%) & & \\
\hline$<12$ months & 6 & $95 \pm 5$ & & 10 & $87 \pm 19$ & 8 & $-10,25$ \\
1 to $<2$ years & 7 & $91 \pm 9$ & & 10 & $88 \pm 8$ & 2 & $-7,11$ \\
2 to $<3$ years & 6 & $95 \pm 3$ & & 9 & $88 \pm 11$ & -9 & $-2,16$ \\
3 to $<4$ years & 4 & $85 \pm 9$ & & 10 & $93 \pm 6$ & -14 & $-17,0$ \\
$4-5$ years & 5 & $78 \pm 20$ & & 9 & $92 \pm 6$ & $-28,-1$ \\
\hline
\end{tabular}

Abbreviation: CI, confidence interval.

Table 4 Spectacle wear compliance for patients in IOL group with 20/40 or better visual acuity with or without stereopsis

\begin{tabular}{|c|c|c|c|c|c|c|}
\hline \multirow[t]{2}{*}{ Age } & \multicolumn{2}{|c|}{ Stereopsis } & \multicolumn{2}{|c|}{ No stereopsis } & \multirow[t]{2}{*}{ Difference in compliance (\%) } & \multirow[t]{2}{*}{$95 \%$ CI of the difference } \\
\hline & No. of eyes & $\%$ of waking hours & No. of eyes & $\%$ of waking hours & & \\
\hline$<12$ months & 6 & $43 \pm 37$ & 5 & $78 \pm 20$ & -34 & $-75,6$ \\
\hline 1 to $<2$ years & 8 & $49 \pm 35$ & 5 & $79 \pm 17$ & -30 & $-63,2$ \\
\hline 2 to $<3$ years & 7 & $58 \pm 32$ & 5 & $88 \pm 6$ & -30 & $-60,0$ \\
\hline 3 to $<4$ years & 5 & $51 \pm 35$ & 5 & $94 \pm 4$ & -43 & $-87,0$ \\
\hline 4 to 5 years & 7 & $67 \pm 36$ & 4 & $89 \pm 15$ & -22 & $-57,14$ \\
\hline
\end{tabular}

Abbreviations: CI, confidence interval; IOL, intraocular lens.

Table 5 Mean hours of daily patching for patients with 20/40 or better visual acuity in their treated eye with or without stereopsis

\begin{tabular}{|c|c|c|c|c|c|c|}
\hline \multirow[t]{2}{*}{ Age } & \multicolumn{2}{|r|}{ Stereopsis } & \multicolumn{2}{|r|}{ No stereopsis } & \multirow[t]{2}{*}{ Difference (h) } & \multirow[t]{2}{*}{$95 \%$ CI of the difference ( $h$ ) } \\
\hline & No. of eyes & Mean hours of daily patching & No. of eyes & Mean hours of daily patching & & \\
\hline$<12$ months & 15 & $3.44 \pm 1.28$ & 15 & $4.81 \pm 1.16$ & -1.37 & $-2.28,-0.45$ \\
\hline 1 to $<2$ years & 15 & $3.17 \pm 1.58$ & 15 & $5.28 \pm 1.02$ & -2.05 & $-3.05,-1.05$ \\
\hline 2 to $<3$ years & 15 & $3.04 \pm 1.47$ & 15 & $5.72 \pm 0.68$ & -2.68 & $-3.55,-1.81$ \\
\hline 3 to $<4$ years & 15 & $2.83 \pm 1.89$ & 15 & $6.25 \pm 1.48$ & -3.41 & $-4.71,-2.10$ \\
\hline 4 to 5 years & 15 & $1.79 \pm 1.73$ & 15 & $5.89 \pm 1.44$ & -4.10 & $-5.32,-2.88$ \\
\hline
\end{tabular}

Abbreviation: $\mathrm{CI}$, confidence interval. 
$20 / 40$ or better acuity and stereopsis. Other factors such as age at cataract surgery, private $v$ s public insurance, and type of cataract were not significantly correlated with having both good visual acuity and stereopsis.

Stereopsis first appears in infants between ages 3 to 4 months and continues to develop over the next few years. ${ }^{18,19}$ Ocular alignment and good visual acuity in both eyes are necessary for the development of highgrade stereopsis. ${ }^{17,20}$ Stereopsis has been shown to improve after correction of refractive errors with glasses and after part-time occlusion therapy in amblyopic children. ${ }^{21-23}$ Most children do not develop stereopsis after unilateral congenital cataract surgery. $5,24,25$ The absence of stereopsis in these children is probably related to manifest strabismus or a poor visual acuity outcome. Bothun et al ${ }^{13}$ has reported that $80 \%$ of infants who undergo unilateral cataract surgery develop strabismus. In this study, we explored the role of patching in the development of stereopsis in children after unilateral congenital cataract surgery.

Many different patching regimens have been proposed after unilateral congenital cataract surgery. Some clinicians have recommended fixed patching regimens, while others have used customized regimens based on the visual acuity of the treated eye. ${ }^{26}$ In preverbal children, visually evoked potentials have been used to customize patching regimen. ${ }^{5,27}$ The IATS protocol specified that all children enrolled in the study should be patched for $1 \mathrm{~h}$ per day per month of life until 8 months of age, after which they were to be patched for $50 \%$ of their waking hours every day or full-time every other day until age 5 years. Patching adherence was monitored every 3 months using 7-day diaries and telephone interviews. We have previously reported that, on average, better visual outcomes are associated with greater amounts of reported patching. ${ }^{8}$ However, in this paper we report that, on average, among children with good vision, those with stereopsis were patched less than children with similar visual acuity for whom there was no evidence of stereopsis. There was no provision in the IATS patching protocol to modify patching regimens based on the acuity of the treated eye. Therefore, it is possible that some study patients developed stereopsis as a result of fewer hours of occlusion. Alternatively, children with stereopsis may have been more resistant to patching therapy because patching deprived them of binocular depth cues. As all of the patients in the cohort we studied had good visual acuity in both eyes, the visual acuity of the treated eye was not likely to be a factor influencing patching adherence.

We have previously reported that children with good visual acuity outcomes after unilateral congenital cataract surgery were patched more than children with poor visual acuity outcomes. ${ }^{28}$ While this finding may appear to be paradoxical with the findings reported in this study, one possible explanation would be that a certain amount of patching is necessary to achieve good visual acuity after unilateral congenital cataract surgery. ${ }^{29}$ Furthermore, this threshold may differ for each child. However, it may be detrimental to a child's stereopsis to be patched more than this threshold amount. The existence of a threshold for patching would be consistent with our observation that similar visual outcomes were achieved with widely varying amounts of patching. ${ }^{8}$

Others have also reported that reduced patching regimens are associated with better stereopsis after unilateral congenital cataract surgery. Jeffery et $a l^{11}$ compared stereopsis for children who underwent intensive ( $80 \%$ of waking hours) vs reduced (25-50\% of waking hours) patching after unilateral congenital cataract surgery during infancy. Visual acuity outcomes were similar in both groups, but a higher percentage of children in the reduced patching group had stereopsis or fusion (50\% vs $14 \%$ ). Lambert ${ }^{30}$ reported $20 / 20$ visual acuity in both eyes and $120 \mathrm{~s} /$ arc of stereopsis in a 11-year-old child who underwent unilateral cataract surgery at age 6 weeks who was treated with a reduced patching regimen. Gregg and Parks ${ }^{9}$ reported highgrade stereopsis in a 8-year-old child who was patched using a progressive patching regimen of $1 \mathrm{~h}$ per day per month of life during the first 8 months of life after unilateral congenital cataract surgery. Wright et al ${ }^{10}$ used a similar patching regimen and reported that 3 of $13(23 \%)$ children developed stereopsis. The IATS patching protocol was similar to the patching regimen used by Greg and Parks ${ }^{9}$ and Greg et al. ${ }^{10}$ However, our findings suggest that this patching regimen likely exceeds the patching needed to achieve good vision and it may interfere with the development of stereopsis. While Greg and Parks ${ }^{9}$ used a progressive patching regimen until age 8 months, patching was maintained at $8 \mathrm{~h}$ per day until age 2 years and then increased to $10 \mathrm{~h}$ per day until age 3 years, after which it was tapered to $8 \mathrm{~h}$ per day to age 5 years and then $6 \mathrm{~h}$ per day to 8 years. Wright reported patching children up to $50 \%$ of their waking hours from age 4 to 12 months and then up to $80 \%$ of their waking hours thereafter. In the IATS, lesser amounts of patching appeared to be sufficient to preserve good visual acuity and stereopsis after the first year of life.

Several randomized clinical trials have reported equivalent visual outcomes for children with strabismic and/or anisometropic amblyopia treated with reduced vs intensive patching regimens. ${ }^{31-33}$ Holmes et al ${ }^{32}$ reported that prescribing $6 \mathrm{~h}$ of patching was just as effective as 
prescribing full-time patching in treating severe amblyopia. Repka et $a l^{33}$ noted that prescribing $2 \mathrm{~h}$ of patching was as effective as prescribing $6 \mathrm{~h}$ of patching in treating moderate amblyopia in children 3 to $<7$ years of age. The lack of correlation between daily patching duration and stereopsis in these studies may reflect the later age of onset of their amblyopia and their older age at the time of treatment. ${ }^{23}$ In a meta-analysis of children from the Amblyopic Treatment Studies with anisometropic amblyopia, Wallace et $a l^{23}$ noted worse stereopsis in children 3 to $<5$ years of age compared with older children that they attributed to their poor cooperation or their inability to understand stereopsis testing.

There were a number of limitations to our study. First, a relatively small number of children had both good visual acuity and a stereopsis response reducing the power of our findings. This is exacerbated by the number of independent variables that we considered. Second, while 15 children had good vision and a stereopsis response, there were inconsistencies in their stereopsis responses. Only three patients had a stereopsis response using all three tests of stereopsis and four additional patients using two tests of stereopsis. It is possible that some stereopsis responses were false-positive responses, particularly in the three patients who had angles of strabismus $\geq 6$ PD. ${ }^{17}$ However, it is also possible that these children had intermittent deviations that may not have been present when stereopsis was tested. Additional children may have had stereopsis that we were unable to detect because of poor patient cooperation related to their young age. It is also possible that some of the children without stereopsis responses who had strabismus may have had stereopsis if their eyes were surgically aligned. However, this is unlikely given the longstanding duration of their strabismus. ${ }^{34}$ It is also difficult to ascertain whether more intensive patching caused manifest strabismus with a resultant loss of stereopsis or the loss of stereopsis from more patching resulted in manifest strabismus. A strength of the study was its design as a randomized clinical trial with protocols for cataract surgery, follow-up examinations, and patching regimens. We also monitored patching adherence at regular intervals to age 5 years using two different techniques -caregiver diaries and telephone interviews. A final strength of our study was our high retention rate. ${ }^{16}$

In conclusion, among patients who undergo early unilateral cataract surgery and who are compliant with their optical correction, reduced patching from age 1 to 5 years may maximize their chances of developing both good visual acuity and stereopsis. Our study suggests that for these children, $\sim 4$ h per day of patching during the first year of life, $3 \mathrm{~h}$ per day from age 1 to 3 years and $2 \mathrm{~h} /$ day when 4 years of age may optimize their likelihood of achieving both good visual acuity in their treated eye and stereopsis.

Summary

What was known before

- It was known that children could achieve good vision and only rarely stereopsis following unilateral cataract surgery during infancy with part-time patching therapy of the fellow eye. However, the optimal amount of patching therapy has not been established

What this study adds

- We showed that children could achieve good vision and stereopsis with less patching than has usually been prescribed for children following unilateral cataract surgery during infancy

\section{Conflict of interest}

The authors declare no conflict of interest.

\section{Acknowledgements}

This work was supported by National Institutes of Health Grants U10 EY13272, U10 EY013287, UG1 EY025553, UG1EY013272, and, in part, by NIH Departmental Core Grant EY006360 and Research to Prevent Blindness Inc., New York, NY, USA.

\section{Members of the Infant Aphakia Treatment Study Group}

Administrative Units and Participating Clinical Centers Clinical Coordinating Center (Emory University7): Scott R Lambert, MD (Study Chair); Lindreth DuBois, MEd, MMSc, CO, COMT (National Coordinator). Data

Coordinating Center (Emory University): Michael Lynn MS (Director), Betsy Bridgman, BS; Marianne Celano PhD; Julia Cleveland, MSPH; George Cotsonis, MS; Carey DrewsBotsch, PhD; Nana Freret, MSN; Lu Lu, MS; Seegar Swanson; Thandeka Tutu-Gxashe, MPH. Visual Acuity Testing Center (University of Alabama, Birmingham): E Eugenie Hartmann, PhD (Director); Anna K Carrigan, MPH; Clara Edwards. Eye Movement Reading Center (University of Alabama, Birmingham and Retina Foundation of the Southwest, Dallas, TX, USA): Claudio Busettini, PhD; Samuel Hayley; Eleanor Lewis, Alicia Kindred, Joost Felius, PhD. Steering Committee: Scott R Lambert, MD; Edward G Buckley, MD; David A Plager, MD; M Edward Wilson, MD; Michael Lynn, MS; Lindreth DuBois, MEd, MMSc; Carolyn Drews-Botsch, PhD; E Eugenie Hartmann, PhD; Donald F Everett, MA. Rotating: Joost Felius, PhD; Margaret Bozic, CCRC, COA; Ann 
Holleschau, BA. Contact Lens Committee: Buddy Russell, COMT; Michael Ward, MMSc.

Participating Clinical Centers (In order by the number of patients enrolled) Medical University of South Carolina; Charleston, South Carolina (14): M Edward Wilson, MD; Margaret Bozic, CCRC, COA; Carol Bradham, COA, CCRC. Harvard University; Boston, Massachusetts (14): Deborah K Vanderveen, MD; Theresa A Mansfield, RN; Kathryn Bisceglia Miller, OD. University of Minnesota; Minneapolis, Minnesota (13): Stephen P Christiansen, MD; Erick D Bothun, MD; Ann Holleschau, BA; Jason Jedlicka, OD; Patricia Winters, OD; Jacob Lang, OD. Cleveland Clinic; Cleveland, Ohio (10): Elias I Traboulsi, MD; Susan Crowe, BS, COT; Heather Hasley Cimino, OD. Case Western Reserve: Faruk Orge, MD; Megin Kwiatkowski; Beth Colon. Baylor College of Medicine; Houston, Texas (10): Kimberly G Yen, MD; Maria Castanes, MPH; Alma Sanchez, COA; Shirley York, OD; Stacy Malone, COA; Margaret Olfson. Oregon Health and Science University; Portland, Oregon (9): David T Wheeler, MD; Ann U Stout, MD; Paula Rauch, OT, CRC; Kimberly Beaudet, CO, COMT; Pam Berg, CO, COMT.

Emory University; Atlanta, Georgia (9): Scott R Lambert, MD; Amy K Hutchinson, MD; Lindreth Dubois, MEd, MMSc, CO, COMT; Rachel Robb, MMSc, CO, COMT; Marla J Shainberg, CO. Duke University; Durham, North Carolina (8): Edward G Buckley, MD; Sharon F Freedman, MD; Lois Duncan, BS, CO, COMT; BW Phillips, FCLSA; John T Petrowski, OD. Vanderbilt University: Nashville, Tennessee (8): David Morrison, MD; Sandy Owings COA, CCRP; Ron Biernacki, CO, COMT; Christine Franklin, COT. Indiana University, Indianapolis, Indiana (7): David A Plager, MD; Daniel E Neely, MD; Michele Whitaker, COT; Donna Bates, COA; Dana Donaldson, OD. Miami Children's Hospital, Miami, Florida (6): Stacey Kruger, MD; Charlotte Tibi, CO; Susan Vega. University of Texas Southwestern; Dallas, Texas (6): David R Weakley, MD; David R Stager Jr MD; Joost Felius, PhD; Clare Dias, CO; Debra L Sager; Todd Brantley, OD. Data and Safety Monitoring Committee: Robert Hardy, PHD (Chair); Eileen Birch, PhD; Ken Cheng, MD; Richard Hertle, MD; Craig Kollman, PhD; Marshalyn Yeargin-Allsopp, MD (resigned); Cyd McDowell; Donald F Everett, MA. Medical Safety Monitor: Allen Beck, MD.

\section{References}

1 Costenbader FD, Albert DG. Conservatism in the management of congenital cataract. AMA Arch Ophthalmol 1957; 58: 426-430.

2 Ryan SJ, Blanton FM, von Noorden GK. Surgery of congenital cataract. Am J Ophthalmol 1965; 60(4): 583-587.
3 Von Noorden GK, Ryan SI, Maumenee AE. Management of congenital cataracts. Trans Am Acad Ophthalmol Otolaryngol 1970; 74(2): 352-359.

4 Frey T, Friendly D, Wyatt D. Re-evaluation of monocular cataracts in children. Am J Ophthalmol 1973; 76 (3): 381-388.

5 Beller R, Hoyt CS, Marg E, Odom JV. Good visual function after neonatal surgery for congenital monocular cataracts. Am J Ophthalmol 1981; 91(5): 559-565.

6 Birch EE, Stager DR. The critical period for surgical treatment of dense congenital unilateral cataract. Invest Ophthalmol Vis Sci 1996; 37(8): 1532-1538.

7 Hartmann EE, Lynn MJ, Lambert SR, Infant Aphakia Treatment Study G. Baseline characteristics of the infant aphakia treatment study population: predicting recognition acuity at 4.5 years of age. Invest Ophthalmol Vis Sci 2014; 56 (1):388-395.

8 Drews-Botsch C, Cotsonis G, Celano M, Lambert SR. Assessment of adherence of visual correction and occclusion therapy in the Infant Aphakia Treatment Study. Contemp Clin Trials 2016 (in press).

9 Gregg FM, Parks MM. Stereopsis after congenital monocular cataract extraction. Am J Ophthalmol 1992; 114(3): 314-317.

10 Wright KW, Matsumoto E, Edelman PM. Binocular fusion and stereopsis associated with early surgery for monocular congenital cataracts. Arch Ophthalmol 1992; 110(11): 1607-1609.

11 Jeffrey BG, Birch EE, Stager Jr DR, Stager DR Sr, Weakley DR Jr. Early binocular visual experience may improve binocular sensory outcomes in children after surgery for congenital unilateral cataract. J AAPOS 2001; 5(4): 209-216.

12 Hartmann EE, Stout AU, Lynn MJ, Yen KG, Kruger SJ, Lambert SR et al. Stereopsis results at 4.5 years of age in the infant aphakia treatment study. Am J Ophthalmol 2015; 159(1): 64-70 e2.

13 Bothun ED, Lynn MJ, Christiansen SP, Neely DE, Vanderveen DK, Kruger SJ et al. Sensorimotor outcomes by age 5 years after monocular cataract surgery in the Infant Aphakia Treatment Study (IATS). J AAPOS 2016; 20 (1): 49-53.

14 Lambert SR, Buckley EG, Drews-Botsch C, DuBois L, Hartmann E, Lynn MJ et al. The Infant Aphakia Treatment Study: design and clinical measures at enrollment. Arch Ophthalmol 2010; 128(1): 21-27.

15 Lambert SR, Buckley E, Drews-Botsch C, duBois L, Hartmann E, Lynn $\mathrm{M}$ et al. A randomized clinical trial comparing contact lens with intraocular lens correction of monocular aphakia during infany: grating acuity and adverse events at age 1 year. Arch Ophthalmol 2010; 128: 810-818.

16 Infant Aphakia Treatment Study G, Lambert SR, Lynn MJ, Hartmann EE, DuBois L, Drews-Botsch C, et al.

Comparison of contact lens and intraocular lens correction of monocular aphakia during infancy: a randomized clinical trial of HOTV optotype acuity at age 4.5 years and clinical findings at age 5 years. JAMA Ophthalmol 2014; 132(6): 676-682.

17 Leske DA, Holmes JM. Maximum angle of horizontal strabismus consistent with true stereopsis. J AAPOS 2004; 8(1): 28-34.

18 Fox R, Aslin RN, Shea SL, Dumais ST. Stereopsis in human infants. Science 1980; 207(4428): 323-324.

19 Held R, Birch E, Gwiazda J. Stereoacuity of human infants. Proc Natl Acad Sci USA 1980; 77(9): 5572-5574. 
20 Odell NV, Hatt SR, Leske DA, Adams WE, Holmes JM. The effect of induced monocular blur on measures of stereoacuity. J AAPOS 2009; 13(2): 136-141.

21 Richardson SR, Wright CM, Hrisos S, Buck D, Clarke MP. Stereoacuity in unilateral visual impairment detected at preschool screening: outcomes from a randomized controlled trial. Invest Ophthalmol Vis Sci 2005; 46(1): 150-154.

22 Lee SY, Isenberg SJ. The relationship between stereopsis and visual acuity after occlusion therapy for amblyopia. Ophthalmology 2003; 110(11): 2088-2092.

23 Wallace DK, Lazar EL, Melia M, Birch EE, Holmes JM, Hopkins KB et al. Stereoacuity in children with anisometropic amblyopia. J AAPOS 2011; 15(5): 455-461.

24 Pratt-Johnson JA, Tillson G. Unilateral congenital cataract: binocular status after treatment. J Pediatr Ophthalmol Strabismus 1989; 26(2): 72-75.

25 Birch E, O'Connor AR. Stereopsis Outcomes in Children Treated for Congenital Cataracts. In: Lloyd IC, Lambert SR (eds) Congenital Cataract-A Concise Guide to Diagnosis and Management. Springer: Switzerland, 2016, pp 225-233.

26 Brown SM, Archer S, Del Monte MA. Stereopsis and binocular vision after surgery for unilateral infantile cataract. J AAPOS 1999; 3(2): 109-113.

27 Lloyd IC, Dowler JG, Kriss A, Speedwell L, Thompson DA, Russell-Eggitt I et al. Modulation of amblyopia therapy following early surgery for unilateral congenital cataracts. $\mathrm{Br}$ J Ophthalmol 1995; 79(9): 802-806.
28 Drews C, Celano M, Cotsonis G, Hartmann EE, Lambert SR The relationship between occlusion therapy and optotype visual acuity at $41 / 2$ years using data from a randomized clinical trial, the Infant Aphakia Treatment Study. JAMA Ophthalmol 2016; e-pub ahead of print 26 May 2016; doi:10.1001/jamaophthalmol.2016.1365.

29 Lambert SR, Plager DA, Lynn MJ, Wilson ME. Visual outcome following the reduction or cessation of patching therapy after early unilateral cataract surgery. Arch Ophthalmol 2008; 126(8): 1071-1074.

30 Lambert SR. Visual outcomes. In: Lloyd IC, Lambert SR (eds). Congenital Cataract. Springer: Switzerland, 2016, pp 197-208.

31 Pediatric Eye Disease Investigator Group. The course of moderate amblyopia treated with patching in children: experience of the amblyopia treatment study. Am J Ophthalmol 2003; 136: 620-629.

32 Holmes JM, Kraker RT, Beck RW, Birch EE, Cotter SA, Everett DF et al. A randomized trial of prescribed patching regimens for treatment of severe amblyopia in children. Ophthalmology 2003; 110(11): 2075-2087.

33 Repka MX, Beck RW, Holmes JM, Birch EE, Chandler DL, Cotter SA et al. A randomized trial of patching regimens for treatment of moderate amblyopia in children. Arch Ophthalmol 2003; 121(5): 603-611.

34 Fawcett SL, Birch EE. Risk factors for abnormal binocular vision after successful alignment of accommodative esotropia. J AAPOS 2003; 7(4): 256-262. 